\title{
CIRUgía RENAL PERCUTÁNEA CON ACCESO MÍNIMO: MINIPERC.
}

\author{
German de la Torre, W alter De Bonis, Horacio Rey y N orberto Fredoto vich.
}

División Urología. Departamento de Cirugía. Hospital G ral. de agudos "C.G.Durand". Buenos Aires. Argentina.

\begin{abstract}
Resumen.- 0 BJETIVO: Describir los detalles técnicos de la nefrolitotomía percutánea con mínimo acceso, Miniperc, y a nalizar los resultados obtenidos con este procedimiento en pacientes adultos.

MÉTO DO S: Hemos realizado 42 mini-perc en un periodo comprendido entre Agosto del 2002 y Diciembre del 2003. La edad promedio de los pacientes fue de 48 años, (19-62). El procedimiento se llevo a cabo empleando un amplat de $14 \mathrm{Fr}$. El tamaño de la litiasis vario entre $1.5-3.5 \mathrm{~cm}$ en su diámetro mayor $\left(1.5-7 \mathrm{~cm}^{2}\right)$. La litofragmentación se realizo mediante litotricia neumática.

RESULTADO S: La duración promedio del procedimiento fue de 75.2 minutos ( rango entre 60 y 120 ). Todos los
\end{abstract}

pacientes fueron dados de alta a las $24 \mathrm{hs}$. Solo tres pacientes $(7.1 \%)$ tuvieron un descenso del hematocrito en 2 puntos. No se dejo nefrostomía en el postoperatorio. El 95,2 \% de los pacientes quedaron libres de litiasis al mes de la cirugía.

CONCLUSIÓN: LOS resultados obtenidos en nuestra serie nos permiten considerar a la nefrolitotricia percutánea con mínimo acceso (M iniperc) como una nueva alternativa para la remoción de la litiasis renal, con una muy baja morbilidad, disminución del índice de hospitalización, escasa demanda analgésica y sin requerimiento tranfusional.

Palabras clave: Litiasis renal. M ínimo acceso. M ini-Perc.

Summary.- O BJEC TIVES: To describe the technique of the minimal access percutaneous nephrolithectomy, miniperc, and to analyze the results obtained with this treatment in adult patients.

METHODS: We performed 42 minipercs between a ugust 2002 and December 2003. Mean patient age was 48 years (19-62). The procedure was undertaken using a $14 \mathrm{Fr}$ Amplat sheath. Stone size varied from $1.5-3.5 \mathrm{~cm}$ in longest diameter $\left(1.5-7 \mathrm{~cm}^{2}\right)$. Lithofragmentation was performed by pneumatic lithotripsy.

RESULTS: Average procedure time was $75.2 \mathrm{~min}$. (range from 60 to 120). All patients were discharged within 24 hours. 0 nly three patients $(7.1 \%)$ have a hema to crit descent over 2 points. $N$ o nephrostomy tube 
was left for the postoperative time. $95.2 \%$ of the patients were stone free 1 month after surgery.

CONCLUSIONS: O ur results make us consider the minimal access percutaneous nephrolithectomy (M iniperc) a minimal option for the elimination of renal stones, with low morbidity, diminishment of the hospital stays, very low analgesic demand, and no transfusion requirements.

Keywords: Renal stones. M inimal access. M ini perc.

\section{INTRODUCCIÓN}

Cuando en 1976 Fernstom y Johannson realizaron la primer litotricia renal percutánea los urólogos comenzamos a comprender el potencial beneficio de la cirugía renal minimamente invasiva.

Lograr una disminución de la morbilidad, el tiempo de internación y de convalecencia, estimularon el perfeccionamiento de esta técnica basada en el empleo de tractos percutáneos.

El desarrollo de nuevos métodos de fragmentación de las formaciones litiasicas colaboró en la efectividad de este tipo de terapéutica.

El tratamiento de los cálculos renales fue sufriendo transformaciones conjuntamente con los avances tecnológicos. En la últimas dos décadas la nefrolitotricia percutánea (N LP) y la litotricia extracorpórea con ondas de choque (ESWL) han reemplazado casi por completo a la cirugía abierta. (1-3,5). Sin embargo, estos procedimientos no están exentos de un cierto grado de morbilidad significativa.

Como ejemplo del desarrollo de nuevas metodológicas tendientes a disminuir dicha morbilidad, presentamos nuestras experiencia en nefrolitotricias percutáneas con mínimo acceso, mini-perc, para el tratamiento de la litiasis renal. Esta técnica descripta por Jarrett en 1998 (4) (Mini-Percutaneous $N$ ephrolithotomy) se caracteriza por su menor morbilidad, conservando la eficiencia y efectividad en la remoción de cálculos.

$\mathrm{N}$ osotros hemos introducido como variante de la técnica original la ausencia de nefrostomía en el postoperatorio.

\section{OBJETVO}

Describir los detalles técnicos de la MIN I-PERC y analizar los resultados obtenidos con este procedimiento.

\section{MATERIAL Y MÉTODO}

Hemos realizado 42 mini-perc en un período comprendido entre Agosto del 2002 y Diciembre del 2003. La edad promedio de los pacientes fue de 48 años, con un rango entre 19 y 62 .

Fueron intervenidas 18 mujeres $(42.9 \%)$ y 24 hombres (57.1\%).

TABLA I. 42 PACIEN TES. VO LUMEN UTIASICO HASTA $7 \mathrm{~cm}$.

- Tiempo quirúrgico

- Tiempo de internacion

- N efrostomia

- Catéter doble J

- ESW L complementaria

- Libres de litiasis al mes

- Variación del hematocrito

- A nalgesia

- Complicaciones

Pacientes con catéter J

Pacientes sin catéter J
$75.2 \min .(60-120)$

$24 \mathrm{Hs}$.

no

21 pacientes

$21.4 \%$ (9 pacientes)

$95.2 \%$

En 3 pacientes, descendió 2 puntos

Diclofenac $150 \mathrm{mg} / \mathrm{dia}$

Ninguna

3 pacientes, 2 infecciones urinarias

1 fístula renocutanea 
El tamaño de la litiasis oscilo entre 1.5 y 3.5 $\mathrm{cm}$ en su diámetro mayor $\left(1.5-7 \mathrm{~cm}^{2}\right)$.

\section{Preparación del paciente.}

Todos los pacientes fueron sometidos a estudios prequirúrgicos rutinarios que incluyeron a nalítico de sangre y orina, urocultivo y antibiograma, Rx de tórax y árbol urinario, ecografía renovesical y urograma excretor. Algunos pacientes fueron evaluados con tomografía computada, y en un caso fue necesaria la realización de urorresonancia.

Se inició terapéutica antibiótica con quinolonas 24 horas antes del procedimiento. La elección del tipo de antimicrobiano fue consensuada con el grupo infectológico de nuestra institución, sobre la base de análisis retrospectivos. Se indicó ayuno de 10 horas.

Todos los procedimientos se llevaron a cabo en quirófano común, empleando un arco en $\mathrm{C}$ como instrumento de localización radioscópica.

\section{Posición.}

Bajo anestesia general, se coloca al paciente en decúbito dorsal (posición de litotomía) con el objeto de realizar en forma retrógrada la tinción del sistema excretor renal.

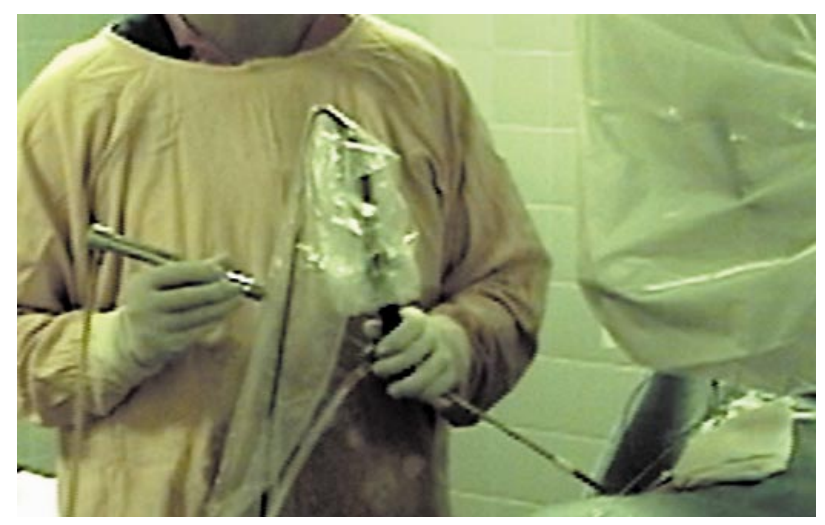

FIG URA 1. Posición quirúrgica y litotricia. O bservamos al paciente en decúbito prono. Se trabaja con amplatz de 14 fr. Empleamos un ureterorrenoscopio semirrigido de $9 \mathrm{fr}$, y litotritor neumático. El procedimiento se realiza bajo guía radioscópica y visualización con endocamara.
Mediante el empleo de un uretrocistoscopio rígido de 20 fr., se identifica el meato ureteral del lado afectado. Bajo control radioscópico, se asciende un catéter ureteral open end de $5 \mathrm{Fr}$. hasta la pelvis renal, para opacificar el árbol urinario superior.

\section{Acceso. Tracto percutáneo.}

Todos los procedimientos se realizaron con el paciente en posición prona.

El acceso percutáneo inicial no presenta diferencias con respecto a la litotricia percutánea standard. Se selecciona el cáliz apropiado para la punción. En la mayor parte de los pacientes de nuestra serie, la punción se realizó a tra vés del grupo calicilar posteroinferior. Se empleó para la misma una aguja de calibre $18 \mathrm{~g}$. Una vez en el interior del sistema colector se introduce un ala mbre guía metálico (PTFE o Hidrofílica). La dilatación se completa con dilatadores fasciales, sobre la guía metálica, hasta 14 fr., dejando por último una camisa de Amplatz de igual calibre.

\section{Nefroscopia. Fragmentación.}

Mediante el empleo de un ureterorrenoscopio de flujo continuo rígido o semirrígido de $9 \mathrm{fr}$., se examina el sistema colector y se identifican las formaciones litiásicas.

Se lleva a cabo la litofragmentación, empleando litotricia neumática, y la extracción de los fragmentos con aspiración (empleado un sistema símil uroback), complementado con el empleo de pinza de 2 ramas. (Figura 1 ).

En algunos pacientes, una vez finalizado el procedimiento, se retira la camisa de amplatz y se deja un catéter $8 \mathrm{fr}$. como nefrostomía hasta que el paciente es extubado.

El tracto urinario fue tutorizado en un grupo de pacientes, con un catéter doble J $4.8 \mathrm{fr}$.

\section{RESULTADOS}

Hemos realizado 42 MINI-PERC cuyos resultados y complicaciones se detallan en la Tabla I.

$\mathrm{N}$ ingún paciente permaneció con nefrostomía en el postoperatorio.

El tiempo de hospitalización fue de un día. El $95.2 \%$ de los pacientes se encontró libre de piedras al mes del procedimiento. 


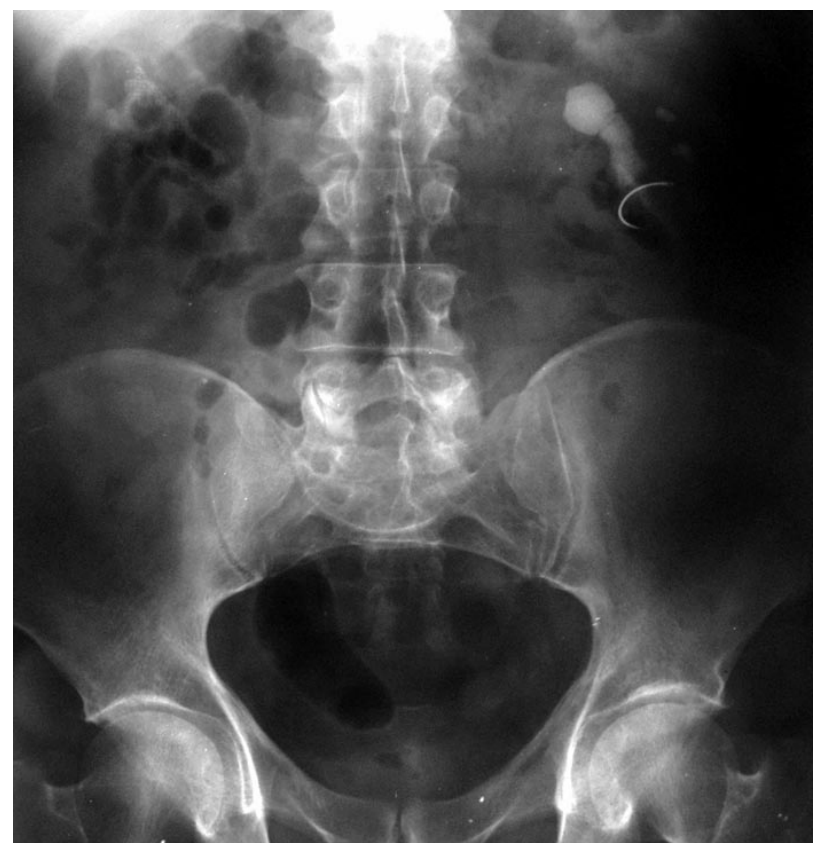

FIG URA 2. Imagen radiográfica. Litia sis pielocalicilar. Paciente monorreno, con antecedente de 2 cirugías renales izquierdas a cielo abierto por litiasis. O bservamos la persistencia de un cuerpo extraño. A mbas condiciones determinaron una ana tomía desfavorable. El empleo de un pequeño canal de trabajo disminuyó las potenciales dificultades técnicas del procedimiento.

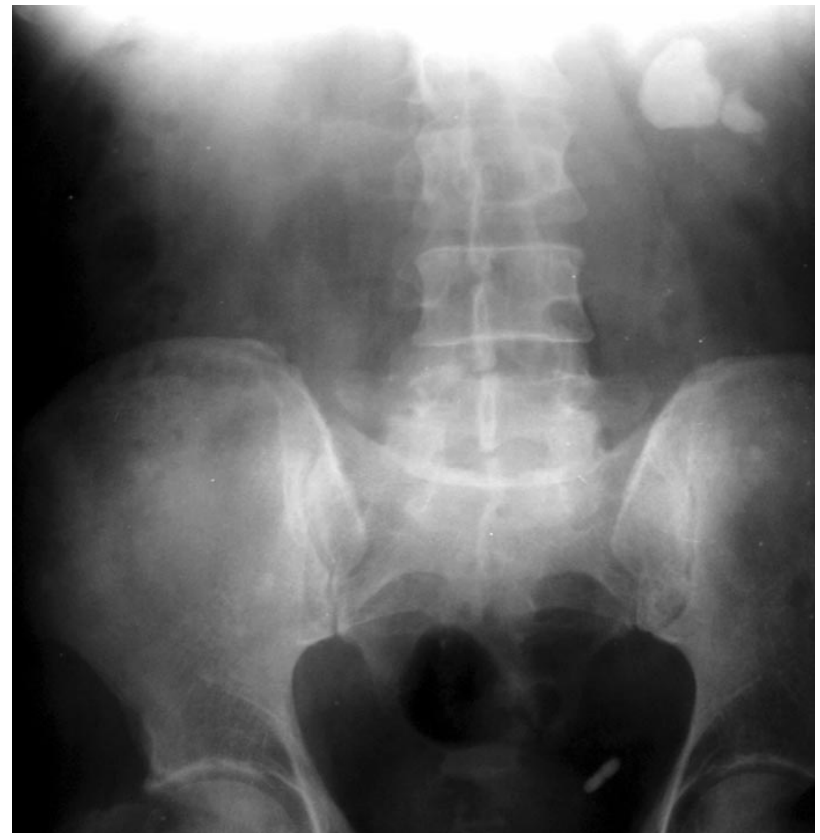

FIG URA 3. Imagen radiográfica. Litiasis pielocalicilar y ureteral distal izquierda. En este caso se realizó ureteroscopia y litotricia endoureteral izquierda, y, a continuación, en el mismo acto quirúrgico, nefrolitotomía con acceso mínimo (M iniperc) pielocalicilar ipsilateral.
En ningún caso fue necesario llevar a cabo tranfusiones. Solo en tres pacientes $(7.1 \%$ de la muestra) se registró variaciones del hematocrito, representada por una caída de dicho índice hematimétrico no mayor a dos puntos; en el resto de la muestra no hubo cambios analíticos del hemograma comparando el pre y el postoperatorio.

La duración promedio del procedimiento fue de 75.2 minutos (rango entre 60 y 120 minutos).

En el $50 \%$ de los pacientes ( 21 pacientes) se coloco un catéter pig tail (doble J), que permaneció una semana. Los 21 pacientes restantes (50\%) permanecieron durante 20 horas con un catéter ureteral recto tipo open end.

Todos los pacientes permanecieron durante 20 horas con sonda vesical.

En 9 pacientes (21.4\%) se completo el tratamiento con ESW L; 7 recibieron una sesión de ESW L, y los restantes 2 sesiones.

En dos pacientes se realizó tratamiento com- binado de litotricia renal y ureteral endoscópica. En uno de ellos en forma bilateral.

Dos pacientes de nuestra serie eran monorrenos, realizándose la cirugía sin mayores inconvenientes.

En un paciente se realizo litotricia renal seguida de endopielotomía anterógrada por estenosis ureteropielica, empleando un electrodo de corte de $3 \mathrm{fr}$.

En 3 pacientes (7.1\%) se presentaron complicaciones, 2 con infección urinaria complicada y 1 con fístula renocutánea; las infecciones urinarias fueron por gérmenes intrahospitalarios (Pseudomona A eruginosa), que requirieron antibioticoterapia endovenosa, a mbos casos se reinternaron, por hipertermia y alteración del estado general, entre los 4 y 5 días posteriores al alta. Se trató de pacientes con diagnostico previo de litiasis renal bilateral, ectasia pielocalicilar, y alteración de la función renal, en los cuales no se había dejado catéter doble J ni nefrostomía en el postoperatorio.

Un paciente, al cual se le había realizado mini-perc izquierda y ureteroscopia bilateral, sin dejar 


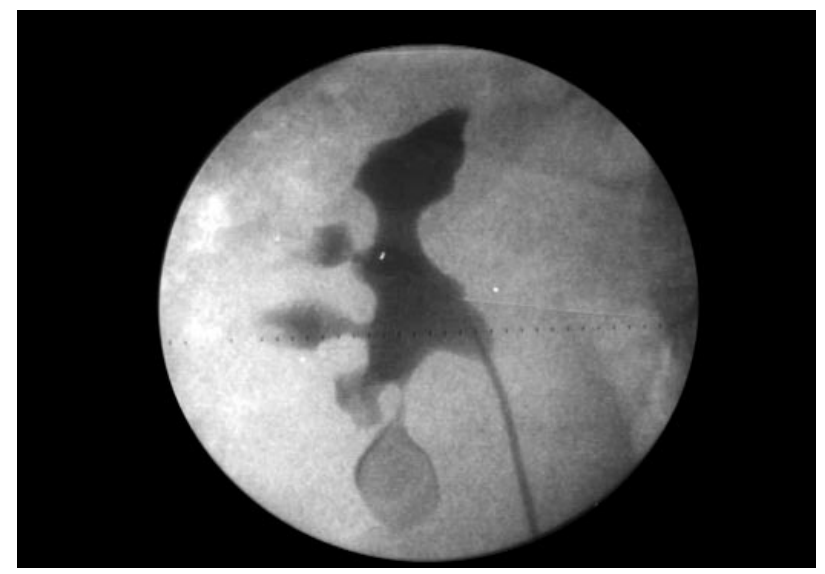

FIG URA 4. Imagen radioscópica. Litiasis en cáliz inferior con ángulo estrecho. Las características del ángulo infundíbulo-pielico y el diámetro infundibular en este caso determinaron la realización de cirugía percutanea con acceso mínimo debido a que dichas variables hubieran dificultado la eliminación de la litiasis mediante el empleo de litotricia extracorpórea por ondas de choque (ESW L).

tutorizada la vía excretora (doble J izquierdo), desarrollo en el riñón izquierdo una fístula renocutánea a las 72 horas de su alta nosocomial por litiasis renal residual, la cual fue resuelta mediante la colocación de un catéter ureteral.

\section{DISCUSIÓN}

Un tracto percutaneo pequeño de 14 fr. fue suficiente para la remoción de los calculo renales en nuestra serie inicial. Sin embargo, en grandes masas litiásicas o tiempo quirúrgico prolongado, su conversión a 26-30 fr. sería de fácil realización. (4).

A diferencia de la serie de Chan y Jarrett (4), donde la dilatación del trayecto percutáneo no supera los 13-14 fr., otros autores describen técnicas de miniperc con dilataciones hasta 22 y 26 fr. (2), no observando diferencias en cuanto al dolor postoperatorio, variación del hematocrito y tiempo de estadía hospitalaria 3 en relación a la percutánea standard de 30 Fr.

Feng y Tamaddon (2), en su análisis prospectivo de la morbilidad vinculada a las técnicas percutáneas, describen trabajos que emplean dilataciones hasta $30 \mathrm{Fr}$, en los cuales se prefiere no dejar nefrostomía en el postoperatorio, permaneciendo el paciente con sonda vesical y catéter pig-tail, nosotros adoptamos igual estrategia, no dejar nefrostomía, pero con dilataciones hasta $14 \mathrm{Fr}$. Vinculado a esta variables, la serie de Bellman y cols. (7) no encuentra diferencias significa tivas entre la nefrolito tomía estándar y la miniperc en lo que respecta a la permanencia del tubo de nefrostomía, sin embargo, en dicho trabajo consideran como mini-tracto, dilataciones hasta 20-26 fr.

En un grupo de nuestros pacientes se dejó tutorizada la vía excretora empleado un catéter doble J, con el objetivo de garantizar la permeabilidad de la vía urinaria y disminuir la presión intracavitaria, lo cual facilita el cierre precoz de la fístula cutánea. En aquellos pacientes en los cuales no se dejo doble J se observaron complicaciones, infección urinaria, fístula persistente y mayor dolor cólico después del alta hospitalaria; consideramos también que en el caso de litiasis obstructiva con ectasia pielocalicilar la permanencia de nefrostomía deberá ser no menor a 24 hs., ya que el tubo de nefrostomía actuaría de drenaje ante una posible perinefritis.

En nuestra serie, ningún paciente requirió tranfusión, presentándose sólo en 3 pacientes modificación del hematocrito.

O bservamos que con el amplatz de 14 fr.no existe distorsión anatómica del cáliz seleccionado como vía de ingreso al sistema excretor, manteniéndose prácticamente indemnes las paredes calicilares e infundibulares, y provocando de esta manera un escaso o nulo sangrado durante el procedimiento. (3).

El $95.2 \%$ de los pacientes se encontraron libres de litiasis al mes de la cirugía. Diferentes resultados son publicados por otros autores (3), quienes observaron un porcentaje de pacientes libres de piedras de un $71 \%$ en igual periodo, mencionando como posible causa el menor campo operatorio. Sin embargo, los resultados obtenidos en nuestra serie son coincidentes con los trabajos de Jarret y colaboradores. (4) Esta técnica nos posibilita una mayor movilidad intrarrenal, con un acceso menos dificultoso hacia los diferentes grupos calicilares. Sin embargo, en nuestra serie sólo 9 pacientes $(21.4 \%)$ requirió como tratamiento complementario la realización de ESW L. La persistencia de restos litiásicos en algunos de estos pacientes se debió a su ubicación dentro de grupos calicilares anteriores de difícil acceso con instrumental rígido o semirrigido, o a que pasaron inadvertidos con la radioscopia intra operatoria. La disponibilidad de instrumental flexible podría haber disminuido sensiblemente el porcentaje de litiasis residual. (6).

O bservamos una disminución en el tiempo de hospitalización. 
Esta técnica permite, además, sin mayores inconvenientes, el tratamiento asociado de la estenosis ureteropiélica.

\section{CONCLUSIÓN}

La cirugía renal percutánea minimamente invasiva en adultos representada por la mini-perc, puede ser considerada como una herramienta que se suma a los métodos ya establecidos en la endourología para el tratamiento de la litiasis renal de tamaño significativo $\left(7 \mathrm{~cm}^{2}\right)$, enriqueciendo el arsenal del endourologista. (Tabla II y Figuras 2-3-4).

La revisión de la literatura y los resultados obtenidos en nuestra serie nos permiten las siguientes consideraciones:

1- Mínimo trayecto percutáneo (14fr.), lo cual contribuye a

2- un menor sangrado,

3- escasa distorsión de la estructura anatómica, y

4- fácil y rápida accesibilidad a la vía urinaria;

5- la ausencia de nefrostomía en el postoperatorio,

6- la menor morbilidad, (3).

7- la rápida reinserción laboral del paciente,y 8- la disminución de los costos vinculado a una precoz externación (3-4), son alguno de los resultados alentadores que permiten considerar a la MINI-PERC como una propuesta terapéutica para el tratamiento de la urolitiasis.

\section{BIBUOGRAFIA y LECTURAS \\ RECOMENDADAS (*lectura de interés y **lectura fundamental)}

*1. JACKMAN, S.; DOCIMO, S.; CADEDDU, J. y cols.: "The mini-perc technique: A less invasive alternative to percutaneous nephrolithotomy". World J. Urol., 16: 371, 1998.

**2. FENG, M.; TAMADDON, K.; BELLMAN, G. y cols.: "Prospective randomized study of various techniques of percutaneous nephrolithotomy". Urology, 58: 345, 2001.

3. PUPPO, P.: "Percutaneous nephrolithotripsy". Current opinion in Urology, 9: 325, 1999.

**4. CHAN, D.; JARRET, T.: "Mini-Percutaneous Nephrolithotomy". J. Endourology, 14: 269, 2000.

5. AL-SHAMMARI, A.; AL-OTAIBI, K.; LEONARD, M. y cols.: "Percutaneous nephrolithotomy in the pediatric population". J. Urol., 162: 1721, 1999.

6. ELBAHANASY, A.; SHALHAV, A.; HOENING, D. y cols.: "Lower caliceal stone clearence after shock wave lithotripsy or ureteroscopy: The impact of lower pole radiographic anatomy”. J.Urol., 159: 676, 1998.

*7. BELLMAN, D.; DAVIDOFF, R.; CANDELA, J.: "Tubeless percutaneous renal surgery". J. Urol., 157: 1578, 1997. 